\title{
Field statistics modelling of beat noise in an optical amplifier
}

\author{
J.T. Kringlebotn \\ K. Blфtekjaer \\ C.N. Pannell
}

\begin{abstract}
The authors present a rigorous and selfcontained noise analysis of an optical amplifier based on the statistics of the input source field and the amplified spontaneous emission field of the amplifier. The method enables us to obtain the frequency dependence of the output electrical noise power, which has not been derived in previous studies of optical amplifier noise based on the photon statistics master equation. The authors characterise for the first time the noise of an optical amplifier in combination with a superfluorescent source, taking into account the frequency dependence of the gain and the presence of optional optical filters.
\end{abstract}

\section{Introduction}

The limiting noise performance of systems incorporating optical amplifiers is normally determined by the amplified spontaneous emission (ASE). Yamamoto [1] used the photon statistics maser equation analysis by Shimoda et al. [2] to analyse the noise and error rate performance of transmission systems incorporating semiconductor amplifiers, and derived an expression for the variance of the photon number rate at the output of the amplifier. This theory has formed a basis for subsequent studies of the subject, including noise characterisation of fibre amplifiers [3].

Most studies of noise in optical amplifiers [1-5] concentrate on systems using single-mode lasers which primarily exhibit phase noise. There is, however, a growing interest in superluminescent diodes and superfluorescent fibre sources for use in optical fibre sensors, especially in fibre optic gyros and white light interferometers. These broadband thermal-like sources exhibit additional AM intensity noise (also called excess noise), which will be amplified and modified by optical amplifiers. We present here, for the first time, a rigorous analysis of this amplified source intensity noise which we will denote as the signalsignal beat noise since the corresponding detector noise

\section{(C) IEE, 1994}

Paper 1082J (E13), first received 4th November 1992 and in revised form 23rd December 1993

J.T. Kringlebotn and K. Bløtekjaer are with the Dept. of Physical Electronics, Faculty of Electronics and Computer Science, Norwegian Institute of Technology, University. of Trondheim, N-7034 Trondheim, Norway

C.N. Pannell is with the Optoelectronics Research Centre, University of Southampton, Southampton SO9 5NH, United Kingdom current is caused by the mixing in the detector (beating) of the randomly phased frequency components of the amplified signal spectrum.

In this paper we present a theoretical noise analysis of a single pass optical amplifier, treating thermal-like sources in particular, but also discussing laser sources. The analysis is primarily concerned with doped fibre amplifiers, but is valid also for semiconductor laser amplifiers. In our analysis the output noise current spectrum is derived from the autocorrelation function of the output detector current by Fourier transform. The autocorrelation function of the output current is obtained by calculating the fourth-order moment of the total optical field at the output detector(s). By using this field statistics method it is straightforward to calculate the frequency dependence of the output noise power. This has not been achieved by the photon statistics master equation analysis used in previous works on optical amplifier noise.

The frequency dependence of the gain is easily accounted for in our analysis, as is also the presence of optional optical filters. It is necessary to include the frequency dependence of the gain when the source is thermal-like, and also when calculating the spontaneousspontaneous beat noise due to beating between the frequency components in the ASE spectrum.

The field statistics method has previously been used to calculate the output electrical noise power spectra of multiple-path fibreoptic systems such as fibreoptic Mach-Zehnder interferometers and recirculating-ring delay lines driven by either lasers [5] or thermal-like sources [6]. Following the lines of this paper, together with the analysis by Tur et al. [5], the field statistics method was recently used to characterise the noise of a recirculating-ring delay line incorporating a fibre amplifier [7] and of a Sagnac interferometer including such a delay line [8].

Our analysis is based on classical field statistics. Loudon and Shepherd [9] used a similar formulation in

One of the authors (JTK) wishes to thank Paul R. Morkel, Richard I. Laming, and David N. Payne, all of the Optoelectronics Research Centre (ORC), University of Southampton, for useful discussions and support. This work was started while the author was working in the ORC as a guest researcher, and it is sponsored by the Royal Norwegian Council for Scientific and Industrial Research. 
a quantum field statistical analysis of optical quantum amplifiers. They derived expressions for the output photon number distribution, but they did not consider the spectral properties of the noise or the influence of a frequency dependent gain.

\section{Electrical beat noise spectrum}

We consider, in the following, a single mode thermal-like input signal field $E_{0}(t)$, with time varying phase and amplitude:

$$
E_{0}(t)=\sqrt{ }\left[i_{0}(t)\right] \exp \left\{j\left[2 \pi v_{0} t+\phi_{0}(t)\right]\right\}
$$

Here, $i_{0}(t)$ is equal to the photocurrent produced when $E_{0}(t)$ is incident on the detector, $v_{0}$ is the centre frequency of the signal spectrum, and $\phi_{0}(t)$ is the phase of the signal field. Both $i_{0}(t)$ and $\phi_{0}(t)$ are random variables. The signal field is assumed to be linearly polarised in a direction which we denote as s-polarised. Fields polarised orthogonal to the signal are denoted as p-polarised.

The output amplified signal field $E_{s}(t)$, is also assumed to be $s$-polarised, and may hence be written as a convolution of the input signal field $E_{0}(t)$ with the transfer function $h_{s}(t)$ representing the gain of the amplifier and any optical filters which may be present:

$$
E_{s}(t)=\int_{-\infty}^{\infty} E_{0}\left(\tau^{\prime}\right) h_{s}\left(t-\tau^{\prime}\right) d \tau^{\prime}
$$

where

$$
h_{s}(t)=\int_{-\infty}^{\infty} H_{s}(v) \exp (j 2 \pi v t) d v
$$

$H_{s}(v)$ is the Fourier transform of $h_{s}(t)$, and has a magnitude

$$
\left|H_{s}(v)\right|=\sqrt{ }[G(v) F(v)]
$$

where $G(v)$ is the amplifier gain spectrum and $F(v)$ is the optional optical filter function. If there is no optical filter, $F(v) \equiv 1$. Similarly, the ASE field in each of the two orthogonal polarisation states ( $s$ and $p$ ) at the output end of the fibre amplifier may be written

$$
\begin{aligned}
& E_{\text {ase }}(t)^{i}=\sqrt{ }\left[i_{\text {ase }}(t)^{i}\right] \exp \left\{j\left[2 \pi v_{p} t+\phi_{\text {ase }}(t)^{i}\right]\right\} \\
& i=s, p
\end{aligned}
$$

where $i_{\text {ase }}(t)^{i}$ is equal to the photocurrent produced when $E_{\text {ase }}(t)^{i}$ is incident on the detector, $v_{p}$ is the peak (or mean) frequency of the ASE spectrum, and $\phi_{\text {ase }}(t)^{i}$ is the random phase of the ASE field. With an optical filter at the output end of the amplifier, the $i$-polarised $(i=s, p)$ output ASE field $E_{n}(t)^{i}$ may be written as a convolution of the inherent ASE field $E_{\text {ase }}(t)^{i}$, with the transfer function $h_{n}(t)$ representing the optical filter:

$$
E_{n}(t)^{i}=\int_{-\infty}^{\infty} E_{\text {ase }}\left(\tau^{\prime}\right)^{i} h_{n}\left(t-\tau^{\prime}\right) d \tau^{\prime} \quad i=s, p
$$

where

$$
h_{n}(t)=\int_{-\infty}^{\infty} H_{n}(v) \exp (j 2 \pi v t) d v
$$

$H_{n}(v)$ is the Fourier transform of $h_{n}(t)$, and has a magnitude

$$
\left|H_{n}(v)\right|=\sqrt{ }[F(v)]
$$

With an infinite detector bandwidth, the total output current is

$$
i(t)=\left\{E_{s}^{*}(t)+E_{n}^{*}(t)^{s}\right\}\left\{E_{s}(t)+E_{n}(t)^{s}\right\}+E_{n}^{*}(t)^{p} E_{n}(t)^{p}
$$

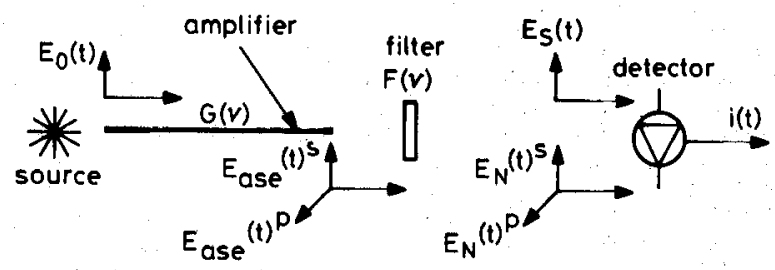

Fig. 1 Schematic drawing of fibre amplifier with gain spectrum $G(v)$ followed by optical filter with filter function $F(v)$

$E_{0}(t)$ and $E_{s}(t)$ are s-polarised input and output signal fields, respectively. $E_{\text {aug }}(t)^{l}$ is $i$-polarised $(i=s, p)$ amplified spontaneous emission (ASE) field at output of fibre amplifier, and $E_{n}(t)^{i}$ is $i$-polarised $(i=s, p)$ ASE field after filtering; $s$ and $p$ represent two orthogonal polarisation states in fibre

To obtain the noise spectrum of the output current, we first calculate the autocorrelation function $\Gamma_{I}(\tau)$ of $i(t)$, which according to Appendix 6 can be expressed as

$$
\begin{aligned}
\Gamma_{I}(\tau)= & \langle i(t+\tau) i(t)\rangle \\
= & \Gamma_{s}^{2}(0)+\Gamma_{s}(\tau) \Gamma_{s}^{*}(\tau)+2 \Gamma_{n}^{2}(0)+2 \Gamma_{n}(\tau) \Gamma_{n}^{*}(\tau) \\
& +2 \Gamma_{s}(0) \Gamma_{n}(0)+\Gamma_{s}(\tau) \Gamma_{n}^{*}(\tau)+\Gamma_{s}^{*}(\tau) \Gamma_{n}(\tau)
\end{aligned}
$$

where

$$
\Gamma_{s}(\tau)=\left\langle E_{s}(t)^{*} E_{s}(t+\tau)\right\rangle
$$

and

$$
\Gamma_{n}(\tau)=\left\langle E_{n}(t)^{i *} E_{n}(t+\tau)^{i}\right\rangle \quad(i=s, p)
$$

are the autocorrelation functions of the output signal and noise field, respectively.

The output electrical noise power spectrum $S(f)$ is given by the Wiener-Khinchine theorem as the Fourier transform $F\left\{C_{I}(t)\right\}$ of the autocovariance function $C_{I}(t)$, which is equal to $\Gamma_{I}(t)$ in eqn. 10 minus the DC terms. We obtain after some manipulations

$$
\begin{aligned}
S(f)= & \int_{-\infty}^{\infty} S_{s}(v) S_{s}(v+f) d v \\
& +\int_{-\infty}^{\infty}\left[S_{s}(v) S_{n}(v+f)^{s}+S_{n}(v)^{s} S_{s}(v+f)\right] d v \\
& +2 \int_{-\infty}^{\infty} S_{n}(v) S_{n}(v+f) d v
\end{aligned}
$$

where

$$
S_{s}(v)=F\left\{\Gamma_{s}(\tau)\right\}=S_{0}(v)\left|H_{s}(v)\right|^{2}=S_{0}(v) G(v) F(v)
$$
and

$$
S_{n}(v)=S_{n}(v)^{s}=S_{n}(v)^{p}=F\left\{\Gamma_{n}(\tau)\right\}=S_{a s e}(v) F(v)
$$

are the photocurrent equivalents of the filtered output optical signal and ASE power spectra, respectively (i.e. the optical power spectra times the responsivity $\rho$ of the detector). Note that the superscripts $s$ and $p$ for the two orthogonal polarisation states will only be used where it is important to emphasise the polarisation state of the ASE field.

Shot noise is not accounted for in the present analysis. It can be included by adding to $S(f)$ a term $S_{\text {shor }}(f)=$ $e\left(\Gamma_{s}(0)+2 \Gamma_{n}(0)\right)$. However, the shot noise is normally 
negligible in systems using optical amplifiers [4], and it will be omitted in the following.

The photocurrent equivalent of the input signal spectrum is given by $S_{0}(v)=\mathbf{F}\left\{\left\langle E_{0}(t)^{*} E_{0}(t+\tau)\right\rangle\right\}$. The photocurrent equivalent of the ASE spectrum corresponding to each of the two polarisation states is given [10] as

$$
S_{\text {ase }}(v)=\rho n_{s p}(v) h v_{p}[G(v)-1]
$$

where $h v_{p}$ is the peak (or mean) ASE photon energy and $n_{s p}(v)$ is the spontaneous emission factor of the amplifier as expressed [10]. With complete population inversion along the amplifier, $n_{s p}(v)=1$.

The first term of the output electrical noise power spectrum $S(f)$ in eqn. 13 represents the signal-signal $(s-s)$ beat noise, and is equal to the expression given [6] in the case of no gain, the second term represents the spontaneous-spontaneous ( $s p-s p)$ beat noise, and the third term represents the signal-spontaneous $(s-s p)$ beat noise. Note that all three noise terms in eqn. 13 may be considered as correlations between optical spectra. The $s-s$ beat noise (excess noise) was included in the variance expression of Yamamoto [1], where he assumed a frequency independent gain. However, this noise was neglected in his, and all subsequent analyses of optical amplifier noise. This is a valid approximation for a single mode laser source, but as we shall see, the $s-s$ beat noise may be the dominant noise contribution when the source is thermal-like. If the source is unpolarised, both the $s-s$ beat noise and the $s-s p$ beat noise terms in eqn. 13 will increase by a factor of 2 , provided that $S_{s}(v)$ is referred to one of the two orthogonal polarisation states.

$S(f)$ in eqn. 13 was calculated for a thermal-like source, but the expressions for the $s-s p$ beat noise and the $s p-s p$ beat noise are also valid for a laser source. The beat noise spectrum in the case of a modulated signal is obtained from eqn. 13 by letting $S_{s}(v)$ represent the (filtered) modulated signal spectrum.

The noise expressions given so far are generally correct for any shape of the signal and gain spectra. However, to obtain explicit expressions for the various beat noise terms, assumptions have to be made about the lineshape of the signal and gain spectra. The amplifier gain spectrum is given by $G(v)=\exp [g(v)]$, where $g(v)$ is the gain parameter, which has a FWHM bandwidth $\Delta v$ and a peak gain frequency $v_{p}$. In the following, we assume that $g(v)$ has a Lorentzian lineshape [11], which is the case for a homogeneously broadened gain medium, and that the signal spectrum has a Gaussian lineshape [11], which is a good approximation for a superfluorescent source [12]. The signal spectrum has a FWHM linewidth $\Delta v_{0}$ and a centre frequency $v_{0}=v_{p}$ (i.e. we consider only the case when the source spectrum is centred at the frequency of maximum gain). In this case, assuming a Lorentzian gain parameter $g(v)$ is a very good approximation provided that $G\left(v_{p}\right) \gg 1$.

We first calculate the frequency dependence of the output electrical noise power, as given by eqn. 13, with and without an optical bandpass filter at the output of the amplifier. We assume an idealised optical filter function

$$
F(v)=\operatorname{Rect}\left[\frac{v-v_{p}}{\Delta v_{f}}\right]
$$

where $\Delta v_{f}$ is the filter bandwidth. With a filter included, the integration limits in eqn. 13 are changed from $-\infty$ and $\infty$ to $v_{p}+f-\Delta v_{f} / 2$ and $v_{p}+\Delta v_{f} / 2$. The positive frequency part of the calculated noise spectra is shown in
Fig. 2, for various values of the filter bandwidth $\Delta \lambda_{f}=\lambda_{p}^{2}$ $\Delta v_{f} / c$. We have assumed an erbium doped fibre amplifier (EDFA) with peak gain wavelength $\lambda_{p}=1532 \mathrm{~nm}$, and a gain parameter bandwidth $\Delta \lambda=\lambda_{p}^{2} \Delta v / c=15 \mathrm{~nm}$.

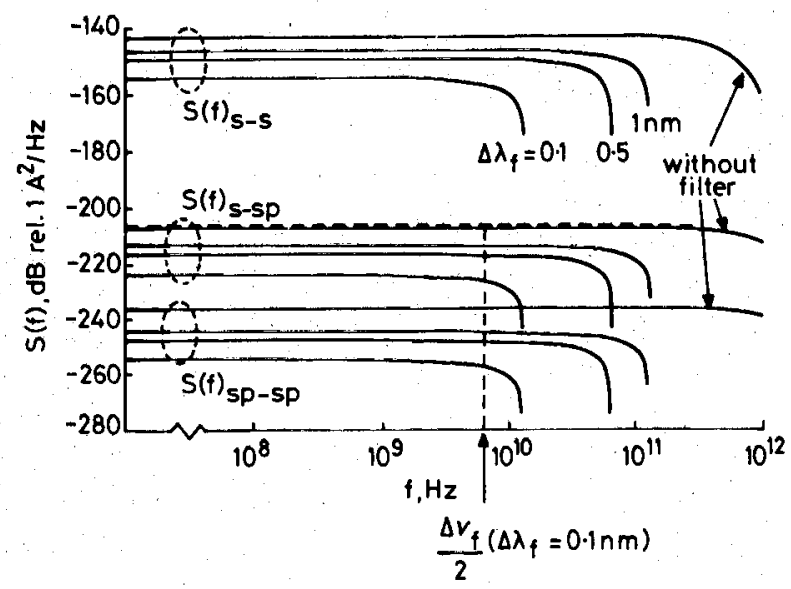

Fig. 2 Positive frequency part of electrical beat noise spectra with superfluorescent source having linewidth $\Delta v_{0}=0.3 \Delta v$ in combination with typical EDFA, with and without optical filtering

Input signal power $P_{0}=0.1 \mathrm{~mW}, G=10 \mathrm{~dB}, \Delta \lambda=15 \mathrm{~nm}, \rho=1 \mathrm{~mA} / \mathrm{mW}$, and $n_{s p}(v)=1$; also shown is $s-s p$ beat noise for single mode laser source with and without filter (dashed lines). The filter bandwidth in this case is $\Delta \lambda_{f}=0.1 \mathrm{~nm}$

Fig. 2 shows that the noise spectra fall off when the electrical frequency $f$ is comparable with the optical linewidths. With an optical bandpass filter with bandwidth $\Delta v_{f}$, the $3 \mathrm{~dB}$ cutoff frequency $f_{3 \mathrm{~dB}}=\Delta v_{f} / 2$, and the spectra go to zero at $f=\Delta v_{f}$.

For comparison, we show the $s-s p$ beat noise spectrum with a single mode laser source (dashed line), which can be obtained from eqn. 13 by assuming that the source linewidth is much smaller than the gain bandwidth. The $s-s p$ beat noise spectrum with a laser source will still have the same cut-off frequency, but with an abrupt cutoff due to the narrow laser spectrum. This is in agreement with Lowery [13], who obtained the electrical beat noise spectra of a laser source in combination with a semiconductor amplifier from the mixing of the spectral components at the input of a square-law photodiode.

Note that, with a single mode laser source and a narrowband optical filter at the output of the amplifier, the measured electrical $s-s p$ beat noise spectrum is a direct measure of the optical filter function, as observed in Fig. 2.

With the thermal-like source, the low frequency noise of all three noise terms are observed to decrease with decreasing filter bandwidth, $\Delta \lambda_{f}$, because this bandwidth is smaller than both the signal and ASE linewidths. Note that, also, the signal is reduced through filtering, and in most cases the signal-to-noise ratio cannot be increased by means of filtering. We will discuss this further in Section 3.4. With a single mode laser source, only the $s p-s p$ beat noise can be reduced at low frequencies by means of an optical filter. Note that, with a single mode laser source, the $s-s$ beat noise is negligible [1], while the $s p-s p$ beat noise is the same as with a thermal-like source.

\section{Electrical beat noise power at low frequencies}

$S(f)$ in eqn. 13 describes the frequency dependence of the output electrical noise power. Usually, we are only interested in the noise at frequencies much lower than the optical linewidths, when $S(f) \simeq S(0)$. In this Section we 
discuss the dependence of the low frequency beat noise on the gain and on the ratio,

$$
r=\frac{\Delta v_{0}}{\Delta v}
$$

between the signal linewidth and the gain parameter bandwidth. We also consider the effect of an optical filter, expressed in terms of the ratio

$$
q=\frac{\Delta v_{f}}{\Delta v}
$$

Finally, we calculate the signal to noise ratio, which is the most relevant parameter for describing the effect of noise on a system. The results are directly applicable to a fibreoptic sensor system using a superfluorescent or superluminescent source in connection with an erbium doped fibre amplifier (or a semiconductor laser amplifier). Results for laser sources are obtained as a special case, but these results are well known from earlier work.

We express the total low frequency beat noise power as

$$
\begin{aligned}
\left\langle i_{N}^{2}(t)\right\rangle_{\text {beat }} & \simeq 2 W S(0) \\
& =\left\langle i_{N}^{2}(t)\right\rangle_{s-s}+\left\langle i_{N}^{2}(t)\right\rangle_{s-s p}+\left\langle i_{N}^{2}(t)\right\rangle_{s p-s p}
\end{aligned}
$$

where $W$ is the detection bandwidth. The factor 2 accounts for integration over both positive and negative electrical frequencies. The three terms in eqn. 20 correspond to the first, second and last term in eqn. 13 with $f=0$, and represent the $s-s$ beat noise, $s-s p$ beat noise, and $s p-s p$ beat noise, respectively.

\subsection{Signal-signal beat noise}

Inserting the first term in eqn. 13 into eqn. 20, using the filter function in eqn. 17 and a Gaussian signal spectrum, we obtain for the $s-s$ beat noise power:

$$
\left\langle i_{N}^{2}(t)\right\rangle_{s-s}=\kappa(G, r, q) \tau_{c 0} 2 W\left(\rho P_{0} G\right)^{2}
$$

where $G=G\left(v_{p}\right), P_{0}$ is the input signal power, and $\tau_{c 0}=$ $(2 \ln 2 / \pi)^{1 / 2} / \Delta v_{0}$ is the input signal coherence time. The factor $\kappa(G, r, q)$ expresses the $s-s$ beat noise reduction relative to the hypothetical situation with frequency independent gain $G$, and is given by

$$
\begin{aligned}
\kappa(G, r, q)= & \frac{4 \ln 2}{G^{2}} \int_{0}^{q / r} \exp \left[\frac{2 g\left(v_{p}\right)}{1+(r x)^{2}}\right] \\
& \times \exp \left[-2 \ln 2 x^{2}\right] d x
\end{aligned}
$$

Note that the relative intensity noise corresponding to $\left\langle i_{N}^{2}(t)\right\rangle_{s-s}$ in eqn. 21 is $10 \log \left[\kappa(G, r, q) \tau_{c 0}\right](\mathrm{dB} / \mathrm{Hz})$. In Fig. $3, \kappa(G, r, q)$ is plotted as a function of the gain, with $r=\Delta v_{0} / \Delta v$ as parameter.

The ratios $r=0.3$ and $r=4$ are typical values for an EDFA in combination with a superfluorescent erbium fibre source (SFE) and a superluminescent diode (SLD), respectively. Fig. 3 shows that the $s-s$ beat noise, relative to the noise obtained with a frequency independent gain, decreases with increasing gain. This is due to the gain narrowing associated with increasing gain. This effect becomes more predominant with increased signal linewidth or decreased gain bandwidth. With an ideal optical filter (see eqn. 17) inserted, the gain is approximately constant within the filter bandwidth, and consequently no gain narrowing effect is observed. Insertion of a narrowband filter centred around $v_{p}$ extracts a part of the gain spectrum where the gain is approximately constant, but it also filters out some of the signal power, and the $s-s$ beat noise is consequently reduced, as observed in Fig. 3.

\subsection{Signal-spontaneous beat noise}

Inserting the second term in eqn. 13 into eqn. 20, using eqns. 16 and 17, we obtain for the $s-s p$ beat noise power:

$$
\left\langle i_{N}^{2}(t)\right\rangle_{s \rightarrow p}=\xi(G, r, q) 4 W \rho^{2} P_{0} n_{s p} h v_{p} G(G-1)
$$

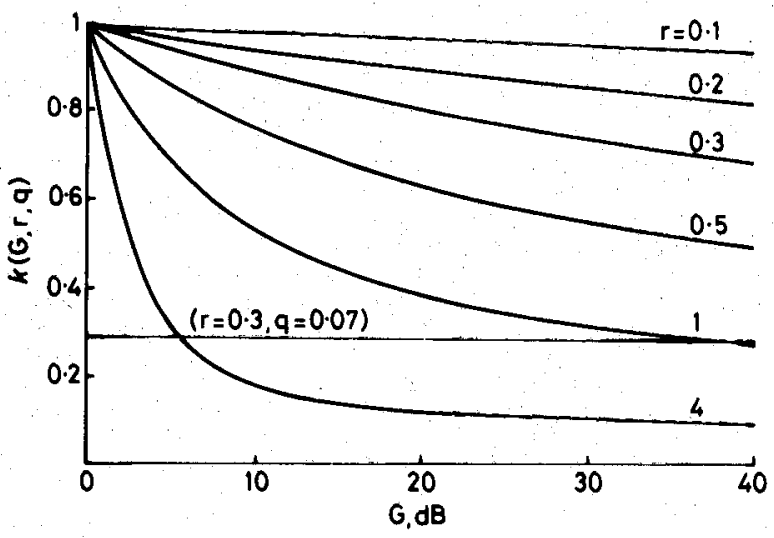

Fig. $3 \kappa(G, r, q)$ for Gaussian signal spectrum, without optical filter $(q \rightarrow \infty)$, as function of amplifier gain $G$, with $r=\Delta v_{0} / \Delta v$ as parameter Also shown is $\kappa(G, 0.3,0.07)$, where, for $\Delta \lambda=15 \mathrm{~nm}$, the optical filter bandwidth $\Delta \lambda_{f}=1 \mathrm{~nm}$

where the factor $\xi(G, r, q)$ expresses the $s-s p$ beat noise reduction relative to the hypothetical situation with frequency independent gain $G$. Here $n_{s p}$ is the effective spontaneous emission factor, which is equal to $n_{s p}\left(v_{p}\right)$ when $G \gg 1[6]$. With a Gaussian signal spectrum,

$$
\begin{aligned}
\xi(G, r, q)= & \frac{2 \sqrt{ }(\ln 2)}{\sqrt{ }(\pi) G(G-1)} \int_{0}^{q / r} \exp \left[\frac{g\left(v_{p}\right)}{1+(r x)^{2}}\right] \\
& \times\left(\exp \left[\frac{g\left(v_{p}\right)}{1+(r x)^{2}}\right]-1\right) \\
& \times \exp \left[-\ln 2 x^{2}\right] d x
\end{aligned}
$$

As for $\kappa(G, r, q)$ in Fig. 3, we plot in Fig. $4 \xi(G, r, q)$ as a function of the gain, with $r$ ranging from 0.1 to 4 .

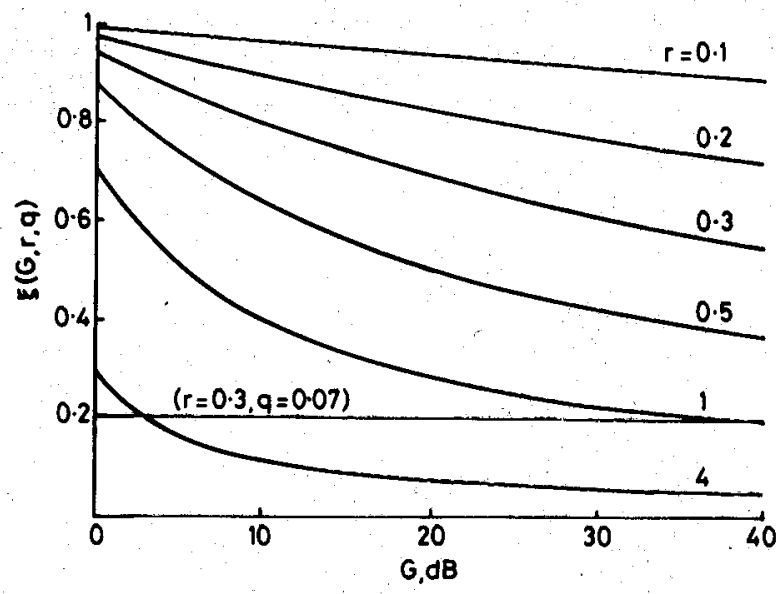

Fig. $4 \xi(G, r, q)$ with Gaussian signal spectrum, without an optical filter $(q \rightarrow \infty)$, as function of amplifier gain $G$, with $r=\Delta v_{0} / \Delta v$ as parameter

Also shown is $\xi(G, 0.3,0.07$, where, for $\Delta \Lambda=15 \mathrm{~nm}$, optical filter bandwidth $\Delta \lambda_{f}=1 \mathrm{~nm}$

Like the $s-s$ beat noise, the $s-s p$ beat noise also decreases with increasing gain and signal linewidth compared to the noise obtained with frequency independent gain. With an optical filter inserted, the wings of the signal and ASE spectra are removed, and therefore the $s-s p$ beat noise is reduced. Since the gain is constant 
within the filter bandwidth, $\xi(G, r, q)$ is independent of the gain.

With a narrow-band single mode laser source the signal gain is constant, and the $s-s p$ beat noise is given by eqn. 23 with $\xi(G, r, q)=1$, in agreement with Reference 4 .

\subsection{Spontaneous-spontaneous beat noise}

Inserting the last term in eqn. 13 into eqn. 20, using eqns. 16 and 17, we obtain for the $s p-s p$ beat noise power:

$$
\left\langle i_{N}^{2}(t)\right\rangle_{s p-s p}=4 W\left[\rho n_{s p} h v_{p}(G-1)\right]^{2} \Delta v_{s p-s p}
$$

where the $s p-s p$ beat noise bandwidth $\Delta v_{s p-s p}$ is given by

$$
\begin{aligned}
\Delta v_{s p-s p} & =\frac{1}{(G-1)^{2}} \int_{-\infty}^{\infty}[G(v)-1]^{2} F^{2}(v) d v \\
& =\frac{\Delta v}{(G-1)^{2}} \int_{0}^{a}\left(\exp \left[\frac{g\left(v_{p}\right)}{1+y^{2}}\right]-1\right)^{2} d y
\end{aligned}
$$

For $F(v)=1$, this is in agreement with the expression given by Laming and Payne [4].

We plot in Fig. $5 \Delta v_{s p-s p}$ relative to $\Delta v$ as a function of the amplifier gain, both with and without optical filtering.

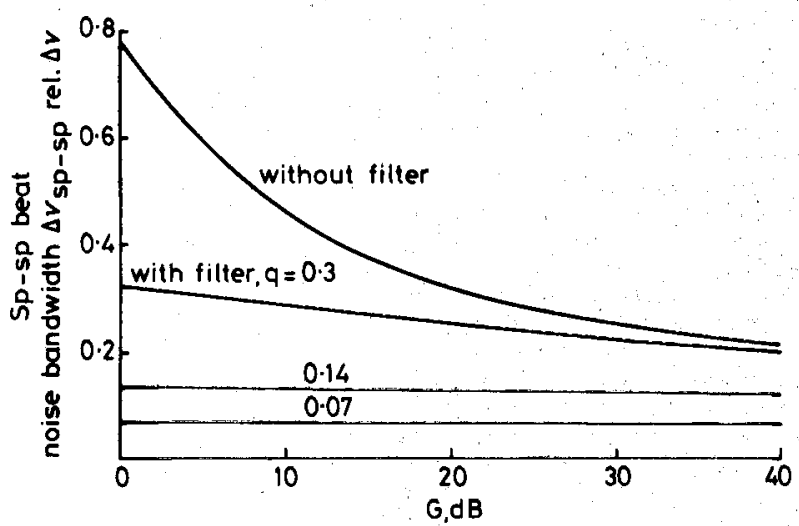

Fig. $6 s p$-sp beat noise bandwidth $\Delta v_{s p-s p}$ plotted as function of amplifier gain $G$ with and without optical fiter relative to FWHM linewidth of gain parameter $g(v)$

In case of $\Delta \lambda=15 \mathrm{~nm}$, filter parameter value of $q=0.07$ corresponds to $\Delta \lambda_{f}=$ $1 \mathrm{~nm}$

Without filter $(q \rightarrow \infty)$ the sp-sp beat noise bandwidth is observed to decrease with increasing gain. This is due to the gain narrowing associated with the increased gain. With a narrow-band optical bandpass filter with constant gain within the filter bandwidth, the $s p-s p$ beat noise bandwidth is equal to the filter bandwidth, as seen from Fig. 5 for $q \simeq 0.07$ and 0.14

\subsection{Signal-to-noise ratio}

In the previous Sections we have discussed the various contributions to the beat noise power at low frequencies. However, we are usually interested in the signal-to-noise ratio, which is defined as

$$
\frac{S}{N}=\frac{\left\langle i_{s}(t)\right\rangle^{2}}{\left\langle i_{N}^{2}(t)\right\rangle}
$$

The mean output signal current $\left\langle i_{s}(t)\right\rangle$ is given by

$$
\left\langle i_{s}(t)\right\rangle=\Gamma_{s}(0)=\int_{-\infty}^{\infty} S_{s}(v) d v
$$

When the beat noise is the dominant noise source, $\left\langle i_{N}^{2}(t)\right\rangle=\left\langle i_{N}^{2}(t)\right\rangle_{\text {beat }}$ in eqn. 20.

Independently of the signal linewidth, it is not possible to increase either $(S / N)_{\text {exc }}$ or $(S / N)_{s-s p}$ by inserting an optical filter. However, $(S / N)_{s p-s p}$ increases with decreasing filter bandwidth, $\Delta v_{f}$, as long as this bandwidth is larger than the signal linewidth $(q>r)$, as illustrated in Fig. 6.

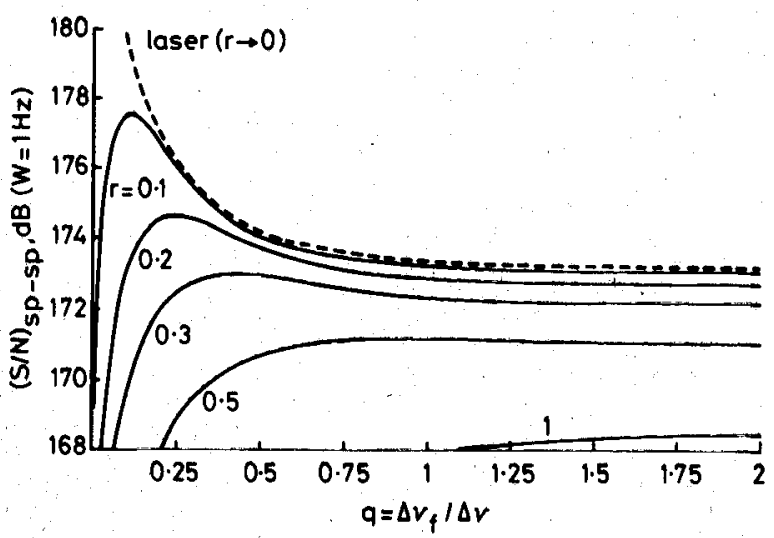

Fig. 6 Signal-to-noise ratio with dominating $s p-s p$ beat noise as function of filter bandwidth $\Delta v_{f}$, for various values of $r=\Delta v_{0} / \Delta v$

$G=10 \mathrm{~dB}, P_{0}=0.1 \mathrm{~mW}, W=1 \mathrm{~Hz}, \Delta \lambda=15 \mathrm{~nm}, n_{a p}=1$ and $\rho=1 \mathrm{~mA} / \mathrm{mW}$

The signal power dependence is different for the three signal-to-noise ratios mentioned above. In Fig. 7 the total signal-to-noise ratio is plotted as a function of $P_{0}$ for

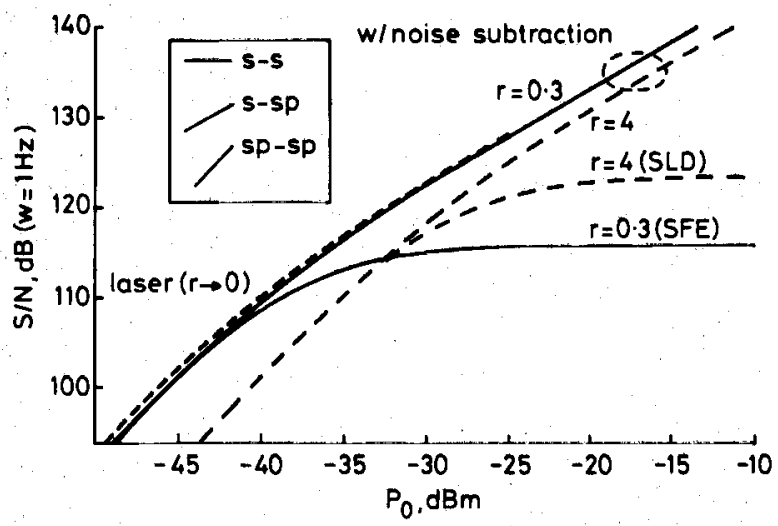

Fig. 7 Signal-to-noise ratio as function of input signal power $P_{0}$ for three different sources

Superfiuorescent erbium doped fibre source (SFE), SLD, and laser source, in combination with an EDFA with gain $G=10 \mathrm{~dB}$ and $n_{3 p}=1$; noise subtractuion is assumed to eliminate $s-s$ beat noise; inset indicates slope of $S / N$ as function of $P_{0}$ when limited by the $s-s$ beat noise, $s-s p$ beat noise and $s p-s p$ beat noise, respectively; detection bandwidth is $1 \mathrm{~Hz}$

three possible sources in combination with an EDFA: an SFE with $\Delta \lambda_{0} \simeq 5 \mathrm{~nm}[14](r=0.3)$, an SLD with $\Delta \lambda_{0} \simeq$ $60 \mathrm{~nm}(r=4)$, and a single mode laser source where $r \simeq 0$. In the latter case the $s-s$ beat noise is negligible, as mentioned in Section 2. Also shown is the signal-to-noise ratio for $r=0.3$ and 4 with $s-s$ beat noise subtraction [15], in which case the $s-s$ beat noise can be reduced by as much as four orders of magnitude by means of a simple referencing scheme. This is done by dividing the signal light equally onto two detectors, where the output from the detectors is applied to a noise subtraction circuit.

Fig. 7 shows that the signal-to-noise ratio is maximum with a laser source. The noise in this case is dominated by the $s p$-sp beat noise for $P_{0}$ less than $n_{s p} h v_{p} \Delta v_{s p-s p}$ $(\simeq-36 \mathrm{dBm})$, in which case the output signal power equals the output ASE power, while the s-sp beat noise dominates for higher input powers. The signal-to-noise ratio obtained by using a typical SFE with $r=0.3$ will be close to that of a laser when the s-s beat noise is subtracted. Without noise subtraction the $s-s$ beat noise will 
dominate for $P_{0}$ higher than about $-35 \mathrm{dBm}$. With an SLD $(r=4)$, much of the signal power spectrum will lie outside the amplifier bandwidth, and is not amplified. Therefore, the signal-to-noise ratio will be lower than for an SFE, except when the noise is dominated by excess noise. In this case the signal-to-noise ratio is proportional to the signal linewidth [14], and an SLD will produce less excess noise than an SFE.

For $G \gg 1$, all three signal-to-noise ratios decrease only slowly with increasing gain. Hence, the curves in Fig. 7 for $G=10 \mathrm{~dB}$ are representative also for higher gains.

\section{Conclusions}

Most theories of noise in optical amplifiers are based on the photon statistics master equation. In this paper, we have presented an alternative approach, based on the statistics of the optical signal and ASE fields. This method has enabled us to obtain the frequency dependence of the output electrical noise power, and it is shown that the electrical noise power spectrum may be substantially modified at $\mathrm{GHz}$ frequencies by the use of narrowband optical filters.

Single mode lasers are used in fibre-optic communication systems with optical amplifiers, and previous noise analyses have been limited to such sources. Our analysis deals primarily with thermal-like sources, such as superluminescent diodes and superfluorescent fibre sources. These are of interest for applications in various kinds of fibreoptic sensors. The main modification introduced by such sources is that $s-s$ beat noise may be the dominant noise contribution for a thermal-like source, whereas it is virtually absent for a laser source. We have presented a complete analysis including the effects of the frequency dependence of the gain and optional optical filtering. Following the lines of this paper, the field statistics method can be used to analyse multiple path optical systems involving optical amplifiers.

\section{References}

1 YAMAMOTO, Y.: 'Noise and error rate performance of semiconductor laser amplifiers in PCM-IM optical transmission systems', IEEE J., 1980, QE-16, pp. 1073-1081

2 SHIMODA, K., TAKAHASI, H., and TOWNES, C.H.: 'Fluctuations in amplification of quanta with application to maser amplifiers', J. Phys. Soc. Japan, 1957, 12, pp. 686-700

3 OLSHANSKY, R.: 'Noise figure for erbium-doped optical fibre amplifiers', Electron. Lett., 1988, 24, pp. 1363-1365

4 LAMING, R.I., and PAYNE, D.N.: 'Noise characteristics of erbium-doped amplifier pumped at $980 \mathrm{~nm}$ ', IEEE Photonics Technol. Lett., 1990, 2, pp. 418-421

5 TUR, M., MOSLEHI, B., and GOODMAN, J.W.: 'Theory of laser phase noise in recirculating fiber-optic delay lines', IEEE J., 1985, LT-3, pp. 20-30

6 TUR, M., SHAFIR, E., and BLØTEKJAER, K.: 'Source-induced noise in optical systems driven by low-coherence sources', IEEE $J$, 1990, LT-8, pp. 183-189

7 KRINGLEBOTN, J.T., and BLØTEKJAER, K.: 'Noise analysis of an amplified fiber-optic recirculating-ring delay line', IEEE $J$., 1994, LT-12, pp. 573-582

8 KRINGLEBOTN, J.T., BLØTEKJAER, K., and PANNELL, C.N.: 'Sagnac interferometer including a recirculating ring with an erbium-doped fibre amplifier'. Proceedings of the 8th optical fiber sensors conference, Monterey, CA, 1992, pp. 6-9

9 LOUDON, R., and SHEPHERD, T.J.: 'Properties of the optical quantum amplifier', Optica Acta, 1984, 31, pp. 1243-1269
10 DESURVIRE, E.: 'Spectral noise figure of $\mathrm{Er}^{3+}$-doped fiber amplifiers', IEEE Photonics Technol. Lett., 1990, 2, pp. 208-210

11 GOODMAN, J.W.: 'Statistical optics' (Wiley, New York, 1985), p. 165

12 DIGONNET, M.J.F., and LIU, K.: 'Analysis of a $1060 \mathrm{~nm}$ $\mathrm{Nd}: \mathrm{SiO}_{2}$ superfluorescent fiber laser', IEEE J., 1989, LT-7, pp. 1009-1015

13 LOWERY, A.J.: 'Transmission-line laser modelling of semiconductor laser amplified optical communications systems', IEE Proc. J, Optoelectr., 1992, 139, pp. 180-188

14 IWATSUKI, K.: 'Excess noise reduction in fiber gyroscope using broader spectrum linewidth er-doped superfluorescent fiber laser', IEEE Photonics Technol. Lett., 1991, 3, pp. 281-283

15 MORKEL, P.R., LAMING, R.I., EDWARDS, H.O., and PAYNE, D.N.: 'Elimination of excess photon noise from fibre super-radiant sources'. Proceedings of the conference on lasers and electrooptics (CLEO), Anaheim, CA, 1990, p. 154

16 GOODMAN, J.W.: 'Statistical optics' (Wiley, New York, 1985), p. 109

\section{Appendix}

\subsection{Autocorrelation function of output current}

The autocorrelation function $\Gamma_{I}(\tau)$ of the output detector current $i(t)$ is, when assuming an s-polarised signal, and an unpolarised ASE field:

$$
\begin{aligned}
\Gamma_{I}(\tau)= & \langle i(t+\tau) i(t)\rangle \\
= & \left\langle\left[\{ E _ { s } ^ { * } ( t + \tau ) + E _ { n } ^ { * } ( t + \tau ) ^ { s } \} \left\{ E_{s}(t+\tau)\right.\right.\right. \\
& \left.\left.+E_{n}(t+\tau)^{s}\right\}+E_{n}^{*}(t+\tau)^{p} E_{n}(t+\tau)^{p}\right] \\
& \times\left[\left\{E_{s}^{*}(t)+E_{n}^{*}(t)^{s}\right\}\left\{E_{s}(t)+E_{n}(t)^{s}\right\}\right. \\
& \left.\left.+E_{n}^{*}(t)^{p} E_{n}(t)^{p}\right]\right\rangle
\end{aligned}
$$

After expanding and averaging $\Gamma_{I}(\tau)$ in eqn. 29 the remaining fourth-order moments are:

$$
\begin{aligned}
\Gamma_{I}(\tau)= & \left\langle E_{s}^{*}(t) E_{s}^{*}(t+\tau) E_{s}(t) E_{s}(t+\tau)\right\rangle \\
& +\left\langle E_{s p}^{*}(t)^{s} E_{s p}^{*}(t+\tau)^{s} E_{s p}(t+\tau)^{s}\right\rangle \\
& +\left\langle E_{s p}^{*}(t)^{p} E_{s p}^{*}(t+\tau)^{p} E_{s p}(t)^{p} E_{s p}(t+\tau)^{p}\right\rangle \\
& +\left\langle E_{s}^{*}(t) E_{s}^{*}(t+\tau)^{s} E_{s p}(t)^{s} E_{s}(t+\tau)\right\rangle \\
& +\left\langle E_{s}^{*}(t+\tau) E_{s p}^{*}(t)^{s} E_{s}(t) E_{s p}(t+\tau)^{s}\right\rangle \\
& +\left\langle E_{s}^{*}(t+\tau) E_{s p}^{*}(t)^{s} E_{s}(t+\tau) E_{s p}(t)^{s}\right\rangle \\
& +\left\langle E_{s}^{*}(t) E_{s p}^{*}(t+\tau)^{s} E_{s}(t) E_{s p}(t+\tau)^{s}\right\rangle
\end{aligned}
$$

With a thermal-like source, the complex Gaussian moment theorem for circular complex Gaussian random processes [16] may be used to reduce the fourth-order moments to second-order moments:

$$
\begin{aligned}
\left\langle u^{*}\left(t_{1}\right) u^{*}\left(t_{2}\right) u\left(t_{3}\right) u\left(t_{4}\right)\right\rangle & = \\
= & \left\langle u^{*}\left(t_{1}\right) u\left(t_{3}\right)\right\rangle\left\langle u^{*}\left(t_{2}\right) u\left(t_{4}\right)\right\rangle \\
& +\left\langle u^{*}\left(t_{1}\right) u\left(t_{4}\right)\right\rangle\left\langle u^{*}\left(t_{2}\right) u\left(t_{3}\right)\right\rangle
\end{aligned}
$$

This theorem also applies for the amplified spontaneous emission (ASE) field from the fibre amplifier. Using eqn. 31 and the fact that the signal field and the ASE field are statistically independent $\Gamma_{I}(\tau)$ may be written as:

$$
\begin{aligned}
\Gamma_{I}(\tau)= & \Gamma_{s}^{2}(0)+\Gamma_{s}(\tau) \Gamma_{s}^{*}(\tau)+2 \Gamma_{s p}^{2}(0)+2 \Gamma_{s p}(\tau) \Gamma_{s p}^{*}(\tau) \\
& +2 \Gamma_{s}(0) \Gamma_{s p}(0)+\Gamma_{s}(\tau) \Gamma_{s p}^{*}(\tau)+\Gamma_{s}^{*}(\tau) \Gamma_{s p}(\tau)
\end{aligned}
$$

where $\Gamma_{s}(\tau)=\left\langle E_{s}^{*}(t) E_{s}(t+\tau)\right\rangle$ and $\Gamma_{n}(\tau)=\left\langle E_{n}^{*}(t)^{i} E_{n}(t\right.$ $\left.+\tau)^{i}\right\rangle(i=s, p)$. 